\title{
Emendation of the Description of Pseudomonas cepacia Burkholder (Synonyms: Pseudomonas multivorans Stainer et al., Pseudomonas kingae Jonsson; EO-1 Group)
}

\author{
HENRY A. SINSABAUGH AND G. W. HOWARD JR.' \\ Food and Drug Administration, Brooklyn, New York 11232, and Biology Department, Adelphi University, \\ Garden City, New York 11530
}

\begin{abstract}
It has been reported that the names Pseudomonas multivorans, $P$. kingae, and $P$. cepacia are synonymous and that $P$. cepacia has priority. We have confirmed this synonymy by subjecting a larger number of strains (15 each) of $P$. multivorans and $P$. kingae to a wider variety of morphological, nutritional, physiological, and biochemical tests than employed in other studies. A total of 127 tests performed on each strain showed that the two groups of strains were $94 \%$ similar, differing only in the following nine characters: growth at $41 \mathrm{C}$; pigmentation; growth on cetrimide agar; nitrate reduction; acid from sucrose and maltose on $\mathrm{O}-\mathrm{F}$ medium; utilization of nicotinate and $n$-dodecane as sole carbon sources; and susceptibility to nalidixic acid. Salient properties for both groups of strains are nutritional versatility and accumulation of poly- $\beta$-hydroxybutyrate as a cellular reserve material.
\end{abstract}

Eugonic oxidizers group number one (EO-1) is a distinct group of nonfluorescent, gram-negative pseudomonads originally collected by $\mathrm{E}$. $O$. King at the Center for Disease Control (CDC), Atlanta, Ga., and characterized by Jonsson (11), who proposed the species name Pseudomonas kingae for this group of bacteria. Strains of the EO-1 group were also described by Gilardi (8), who included a partial nutritional characterization of 10 strains.

Pseudomonas multivorans was first described, but not named, by Morris and Roberts (20), who studied 18 strains isolated from Trinidad soil and river water and cited the following as salient physiological characteristics: accumulation of poly- $\beta$-hydroxybutyric acid (PHB) as a cellular reserve material; utilization of citrate as a sole source of carbon; proteolysis of gelatin and milk; oxidation of numerous carbohydrates; strong lipolysis; hydrolysis of esculin; intense yellow to reddish-purple pigmentation; and polar multitrichous cells. Some of these Trinidad strains and isolates from clinical sources and soil were later studied by Stanier et al. (30), who proposed the name Pseudomonas multivorans for them on the basis of their nutritional, cytological, and biochemical similarities. Stanier et al. also noted that these strains accumulated $\mathrm{PHB}$ and were nutritionally versatile in the type and number of organic compounds utilized as carbon sources.

${ }^{1}$ Present address: Environment 2000 Limited, Denison, Iowa 51442 .
The importance of $P$. kingae and $P$. multivorans as etiological agents of clinical infection has been documented. $P$. kingae strains have been reported as causing necrotizing pneumonitis (7) and bacterial endocarditis $(25,29)$. In addition, $P$. kingae has been isolated from a benzalkonium chloride disinfecting solution which was responsible for numerous cases of urinary tract infection (9). Pedersen et al. (21) examined strains of $P$. multivorans from sputum, a tracheal aspirate, and blood cultures and concluded that they may be etiologically significant in blood infections. As with $P$. kingae, $P$. multivorans strains have been isolated from various disinfectant solutions in the hospital environment and have been responsible for numerous cases of clinical infection $(2,4,19)$. Evidently, both $P$. kingae and $P$. multivorans are opportunistic pathogens potentially hazardous in their ability to contaminate and possibly grow in disinfectant solutions.

Several workers have noted the similarity between $P$. kingae and $P$. multivorans. Pickett and Pedersen (22) compared three strains of $P$. kingae and seven strains of $P$. multivorans on the basis of 52 characteristics and concluded that there was no feature for which one of these groups was uniformly positive and the other uniformly negative. Gilardi (8) also noted this similarity when he compared ten $P$. kingae strains with one strain of $P$. multivorans. However, no formal proposal was made concerning the synonymy of $P$. kingae and $P$. multivorans. 
Ballard et al. (1) established synonymy between $P$. multivorans and the phytopathogen $P$. cepacia Burkholder (5) on the basis of deoxyribonucleic acid homology and other characteristics. Snell et al. (28) examined morphological, biochemical, serological, and other properties of three strains- of EO-1, the type strain of $P$. kingae, and two strains of $P$. multivorans (including the type strain) and found them similar to 35 field strains and the type strain of $P$. cepacia. They proposed that members of all of these species are similar and should be designated as $P$. cepacia, the name which has priority. Results obtained in the study reported here support this proposal by confirming the synonymy of $P$. kingae with $P$. multivorans. Fifteen strains of each of these two species, isolated from clinical and environmental sources, were compared on the basis of a wide variety of biochemical and nutritional properties. This report presents the results of a study of a considerably larger number of strains of $P$. kingae and $P$. multivorans than previously studied by other workers. Additional data are presented on resistance of these strains to various antibiotics and on the number and arrangement of their flagella.

\section{MATERIALS AND METHODS}

Bacterial strains. Fifteen strains of $P$. kingae (EO-1) were examined and compared with 15 strains of $P$. multivorans. All $P$. kingae strains were received from R. E. Weaver (CDC, Atlanta, Ga.). The type strain for P. kingae (A977 = ATCC 25416) designated by Jonsson (11) was not included in this study.
Fifteen of the 19 strains of $P$. multivorans, including the type strain (Stanier's \#382 = ATCC 17759) characterized by Stanier et al. (30), were obtained from the American Type Culture Collection. Tables 1 and 2 show designations and sources for all strains used in this study. Initially, cultures were grown at $35 \mathrm{C}$ for 18 to $24 \mathrm{~h}$ in flasks of tryptic soy (Difco) broth on a reciprocal shaker and then streaked on plates of yeast extract (YE) agar. The most prevalent colonial type was picked from these plates to establish stock cultures maintained on slants of YE agar, stored at 4 to $5 \mathrm{C}$, and transferred at 3- to 4-week intervals.

Nutritional (substrate utilization) tests. Nutritional tests were performed essentially as described by

TABLE 1. Strains of P. kingae studied

\begin{tabular}{c|c|l}
\hline Strain no. & CDC no. & \multicolumn{1}{|c}{ Origin } \\
\hline 1 & B3720 & Urine \\
2 & B3586 & $\begin{array}{l}\text { Urine } \\
\text { Benzalkonium chloride } \\
\text { disinfectant } \\
\text { Baby lotion (with hex- } \\
\text { achlorophene) }\end{array}$ \\
& B3619 & Blood \\
4 & B2118 & Cerebral spinal fluid \\
5 & B1805 & Lymph node \\
6 & A9894 & Baby lotion (with hex- \\
7 & B2505 & achlorophene) \\
8 & B1731 & Lung \\
& & Urine \\
9 & A8807 & Urine \\
10 & A8653 & Lung \\
11 & A8352 & Urine \\
13 & A8619 & Urine \\
14 & A7734 & Antiseptic solution \\
15 & B1267 & . \\
\hline
\end{tabular}

${ }^{a}$ Assigned for present study.

TABle 2. Strains of Pseudomonas multivorans studied ${ }^{a}$

\begin{tabular}{c|c|c|l}
\hline $\begin{array}{c}\text { Strain } \\
\text { no. }\end{array}$ & $\begin{array}{c}\text { ATCC } \\
\text { no. }\end{array}$ & $\begin{array}{c}\text { Serial } \\
\text { no. }\end{array}$ & \multicolumn{1}{|c}{ Origin and other designations $^{\alpha}$} \\
\hline 16 & 17460 & 85 & River water, Trinidad; B2320 \\
17 & 17478 & 104 & Soil, California (D-mandelate enrichment at 30 C) \\
18 & 17616 & 249 & Soil, California (anthranilate enrichment at 41 C); strain S-4-AN \\
19 & 17759 & 382 & Soil, Trinidad (isolate 8); Sneath D335; NCIB 9085; type strain \\
20 & 17760 & 383 & Forest soil, Trinidad (isolate 61); Sneath D336; NCIB 9086 \\
21 & 17761 & 384 & Rotting tree trunk, Trinidad (isolate 7c); Sneath D337; NCIB 9087 \\
22 & 17763 & 386 & Baby's thermometer, Bristol Royal Infirmary \\
23 & 17765 & 396 & Urine (child), Bristol Royal Infirmary; B2394 \\
24 & 17766 & 397 & Urine (child), Bristol Royal Infirmary; B2443 \\
25 & 17769 & 420 & Soil, Trinidad; NCIB 9088; HCC 79 \\
26 & 17770 & 421 & Soil, Trinidad; NCIB 9089; HCC B175 \\
27 & 17771 & 422 & Soil, Trinidad; NCIB 9091; HCC B338 \\
28 & 17772 & 423 & Soil, Trinidad; NCIB 9092; HCC B339 \\
29 & 17773 & 424 & NCIB 9093; HCC B340 \\
30 & 17774 & 425 & Forest soil, Trinidad; Sneath T1 and B269; NCIB 9135 \\
\hline
\end{tabular}

${ }^{a}$ All cultures were purchased from the American Type Culture Collection, Rockville, Md.

${ }^{\circ}$ Assigned for present study.

${ }^{c}$ Assigned by Stanier et al. (30).

${ }^{d}$ Abbreviations: NCIB, National Collection of Industrial Bacteria; HCC, Hankey Culture Collection. 
Stanier et al. (30). Strains were tested for utilization of carbon sources, in both agar and broth media composed of a standard mineral base [Hutner's vitamin-free mineral base $(6) ; 0.08 \mathrm{M}$ potassium phosphate buffer, $\left.\mathrm{pH} 6.8 ; 0.1 \%\left(\mathrm{NH}_{4}\right)_{2} \mathrm{SO}_{4}\right]$ plus 0.1 to $0.2 \%$ (wt/vol) of the carbon source. The agar media contained 1\% (wt/wol) Noble agar (Difco), and plates were inoculated by the replica plating technique (17). Agar tests were repeated four to six times, and the data were analyzed statistically, using Student's $t$ test for determining significant difference (27). Broth media were inoculated with cells grown for 18 to $24 \mathrm{~h}$ at $30 \mathrm{C}$ in $\mathrm{YE}$ broth and rinsed three times with phosphate buffer $\left(\mathrm{KH}_{2} \mathrm{PO}_{4}{ }^{+} \mathrm{Na}_{2} \mathrm{HPO}_{4}, 0.033 \mathrm{M}, \mathrm{pH}\right.$ 6.8). The compounds tested are listed in Table 3.

Physiological and biochemical tests. The following tests were performed as described by Stanier et al. (30): denitrification; nitrate and at mospheric nitrogen as sole nitrogen sources; anaerobic growth in glucose medium; ability to grow at $4,25,37,39$, and $41 \mathrm{C}$ in YE broth; motility; pigment production; aromatic ring (catechol) cleavage; constitutive arginine dihydrolase production; oxidase production (14); gelatin hydrolysis; lecithinase production (the egg yolk reaction); starch hydrolysis; and Tween 80 hydrolysis.

The following tests for the identification of nonfermentative, gram-negative rods were performed as described by King (12): acid production from six different carbohydrates in the O-F medium of Hugh and Leifson (10) and in a peptone broth medium; acidification of $10 \%$ glucose or lactose-phenol red agar slants; and growth on triple sugar-iron agar slants (TSI), MacConkey agar, Salmonella-Shigella agar, Simmons' citrate agar, and cetrimide agar $(0.08 \%$ hexadecyltrimethylammonium bromide). Other tests used were: nitrate reduction; anaerobic growth and acid production in $\mathrm{O}-\mathrm{F}$ glucose medium; pigment production on medium A (pseudomonas "P" agar) and medium B (pseudomonas " $F$ " agar) of King et al. (13), heart infusion tyrosine, TSI, and motility agars; hydrogen sulfide production on TSI agar; esculin hydrolysis; catalase, urease, and indole production; gelatin liquefaction; and methyl red, Voges-Proskauer, and litmus milk reactions. Colonial morphology and hemolytic reaction were determined on blood agar plates (tryptic soy agar and 5\% defibrinated sheep blood; Scott Laboratories, Fiskeville, R.I.) incubated for $48 \mathrm{~h}$ at $37 \mathrm{C}$. All media were from Difco unless otherwise stated.

Cytological observations (cellular morphology). Gram-staining reactions (Hucker's modification) were determined. All strains were examined for flagella arrangement and number by using the modification of Leifson's (18) flagella-staining method described in the CDC Laboratory Methods Manual (15). Flagella numbers were determined by the statistical method of Lautrop and Jessen (16). The accumulation of PHB as a cellular reserve material was determined by phase contrast microscopy of wet mounts as described by Stanier et al. (30). Photomicrographs of flagellated cells and PHB-containing cells were prepared by using a $\times 100$ oil immersion, phase contrast objective (numerical aperture, 1.25).

Antibiotic susceptibility tests. All strains were tested for susceptibility to 17 different antibiotics by using the standardized single-disk diffusion method of Bauer et al. (3) and Ryan et al. (23). All antibiotics were from Difco except for carbenicillin, which was from Baltimore Biological Laboratories, Cockeysville, Md.

\section{RESULTS AND DISCUSSION}

Nutritional tests. The results of carbon-substrate utilization tests performed with both basal agar and basal broth media are listed in Table 3 . The percentages of strains positive in this table have been converted to + (positive), - (negative), or $\mathrm{V}$ (variable) symbols on the basis of the percentage divisions as described by Pickett and Pedersen (22); i.e., $+=85$ to $100 \%$ of strains positive, $\mathrm{V}=16$ to $84 \%$ of strains positive, and - $=0$ to $15 \%$ of strains positive. The growth response patterns of $P$. kingae and $P$. multivorans strains were similar when compared by these percentage divisions. The results for substrate utilization tests performed with the basal agar medium represent responses for four to six trials (usually six) on each substrate. By comparison of the symbols assigned on the basis of the percentage divisions, $P$. kingae and $P$. multivorans showed no difference in growth response on 37 of 46 substrates tested on the basal agar medium. Substrates for which different growth responses were noted (Table 3) were $o$-hydroxybenzoate, $m$-hydroxybenzoate, phenol, acetamide, nicotinate, $n$-butanol, ethanol, $n$-propanol, and $\mathrm{L}(+)$-mandelate. However, for the last four of these substrates, the differences between the percentage of positive $P$. kingae strains and the percentage of positive $P$. multivorans strains was minimal (14 to $27 \%$ difference) and can be excluded as insignificant. When the original raw data (i.e., number of strains positive for any given replica test on a given substrate) for the tests run on agar plates were analyzed statistically by Student's $t$ test, the two sets of strains were not significantly different in ability to utilize 37 of the 46 substrates. The nine substrates for which significant differences $(P<0.01)$ in growth response were noted were $\mathrm{D}(+)$-xylose, $\mathrm{L}(+)$-rhamnose, butyrate, $\mathrm{L}(+)$-mandelate, $o$-hydroxybenzoate, $m$-hydroxybenzoate, phenol, testosterone, and acetamide. Five of these were the same compounds for which differences were found by comparison of the symbols assigned in Table 3.

On the basis of the symbols assigned in Table 3, $P$. kingae and $P$. multivorans showed no difference in growth response on 41 of 49 substrates tested in the basal broth medium. Substrates for which different growth responses were obtained were sucrose, cellobiose, $\mathrm{D}(+)$. 
TABLE 3. Nutritional properties of $P$. kingae and $P$. multivorans ${ }^{a}$

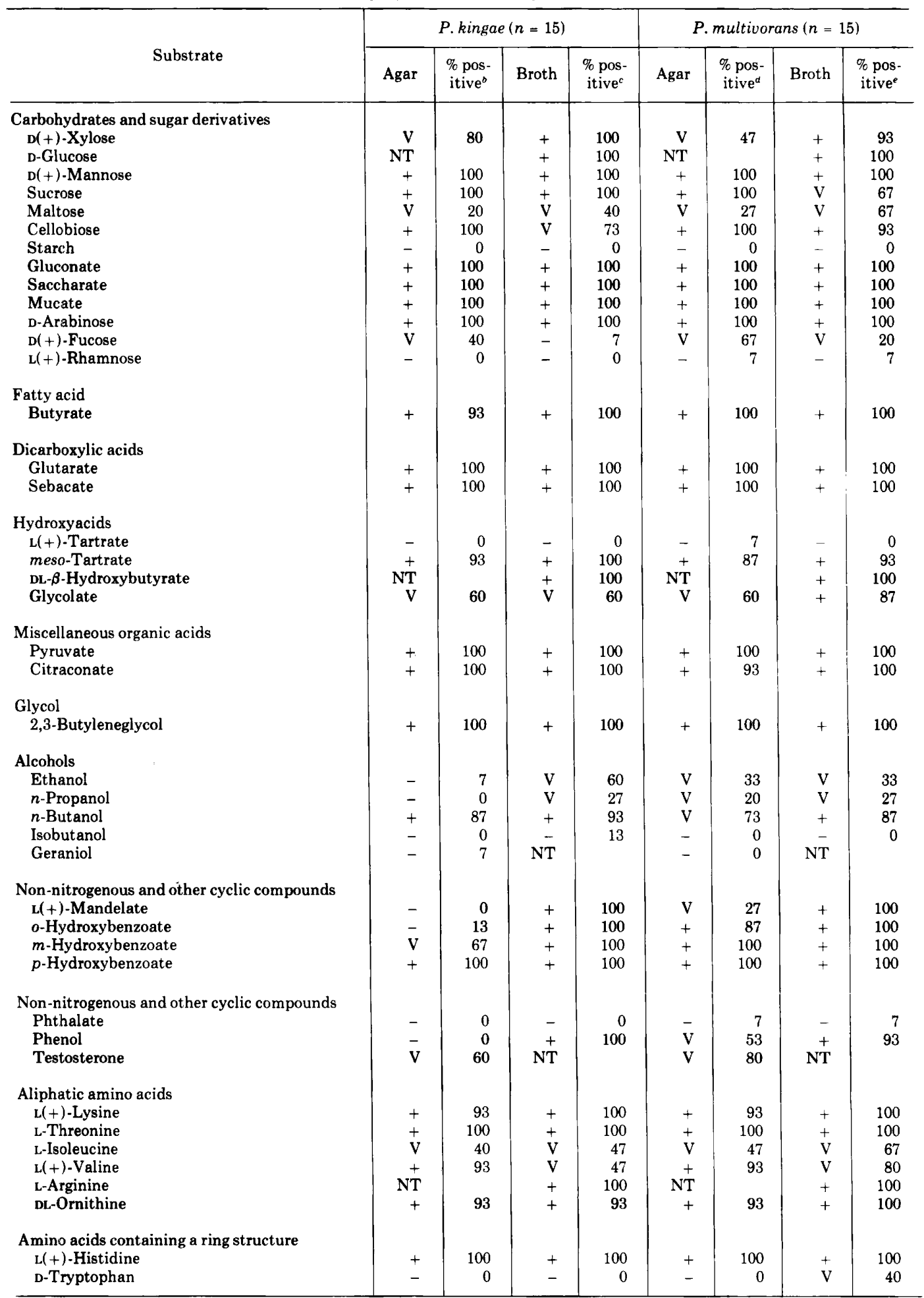


TABLE 3-Continued

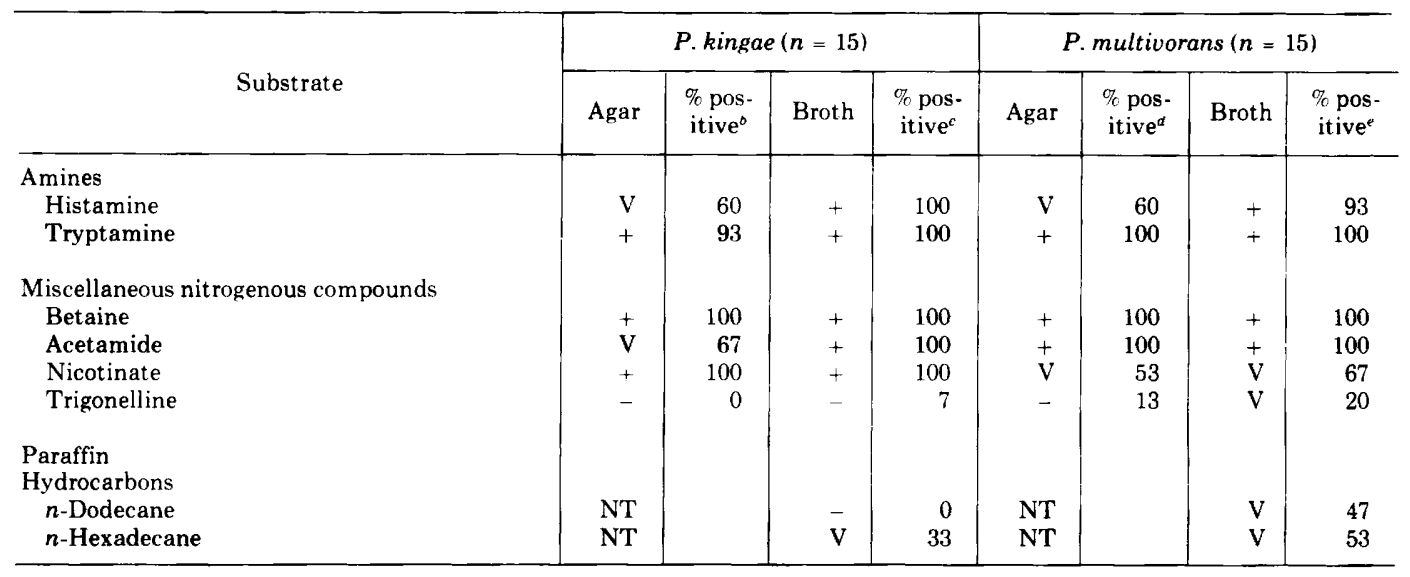

${ }^{a}$ Percent positive indicates the percentage of strains able to utilize the substrate. Single tests were performed for each strain on broth media; four to six replica tests were performed for each strain on agar media. Symbols assigned on the basis of the percentage values:,+ 100 to $85 \%$; V, 84 to $16 \% ;-, 15$ to $0 \%$. NT, Not tested.

'Strain numbers of positive strains for each substrate in agar medium used by a fraction of the strains of $P$. kingae are the following: xylose, all except $5,11,13$; maltose, 2, 6, 11; D-fucose, 2, 4, 5, 9, 12, 14; butyrate, all except 13; meso-tartrate, all except 13; glycolate, all except $4,8,12,13,14,15$; ethanol, $14 ; n$-butanol, all except 11,13 ; geraniol, 14; 0 -hydroxybenzoate, 11 , $14 ; m$-hydroxybenzoate, all except $5,6,9,12,14$; testosterone, all except $4,8,12,13,14,15$; L-lysine, all except 13 ; L-isoleucine, $1,3,6,7,11,15$; L-valine, all except 8 ; DL-ornithine, all except 13 ; histamine, all except $4,5,8,10,12,13 ;$ tryptamine, all except 5 ; acetamide, all except $4.8,12,13,15$.

c Strain numbers of positive strains for each substrate in broth used by a fraction of the strains of $P$. kingae are the following. maltose, $5,6,8,9,10,11$; cellobiose, all except 4,6, 9, 15; D-fucose; 14; glycolate, all except 4, 8, 12, 13, 14, 15; ethanol, all except $5,7,10,11,13,15 ; n$-propanol, $1,2,3,12 ; n$-butanol, all except 13 ; isobutanol, 3,$12 ; \mathrm{L}$-isoleucine, $1,3,8,11,12,13,14 ; \mathrm{L}$-valine, $1,4,8,12,13,14,15$; ol-ornithine, all except 13 ; trigonelline, $14 ; n$-hexadecane, $2,3,7,10,12$.

a Strain numbers of positive strains for each substrate in agar medium used by a fraction of the strains of $P$. multivorans are the following: xylose, 17, 18, 19, 22, 23, 24, 29; maltose, 21, 26, 27, 28; D-fucose, all except 16, 17, 18, 25, 27; L-rhamnose, 25; L-tartrate, 17; meso-tartrate, all except 19, 27; glycolate, all except 16, 18, 21, 23, 24, 30; citraconate, all except 18; ethanol, 17, $22,23,24,29 ; n$-propanol, $17,22,29 ; n$-butanol, all except $18,21,25,27 ;$ L-mandelate, 17, 19, 22, 29; o-hydroxybenzoate, all except 23,24 ; phthalate, 18 ; phenol, all except $18,19,21,22,23,24,28$; testosterone, all except 18 , 23, 24; 1 -lysine, all except 30 ; L-isoleucine, $16,17,22,23,25,28,29 ; \mathrm{L}$-valine, all except 18; DL-ornithine all except 18; histamine, all except 18, 23, 24, 26, 27, 30 ; nicotinate, all except $17,19,20,21,22,26,29$; trigonelline, 18,28

- Strain numbers of positive strains for each substrate in broth used by a fraction of the strains of $P$. multivorans are the following: xylose, all except 28 ; sucrose, all except 16, 18, 20, 25, 30; maltose, all except 16, 17, 18, 23, 24; cellobiose, all except 17; D-fucose, 22, 23, 24; L-rhamnose, 25; meso-tartrate, all except 17; glycolate, all except 23, 24; ethanol, 17, 22, 23, 24, 29; $n$-propanol, $17,22,23,29 ; n$-butanol, all except 18,21 ; phthalate, 18; phenol, all except 28 ; L-isoleucine, all except 18 , 20, 21,24 , 27; L-valine, all except 16, 28, 29; D-tryptophan, 16, 20, 21, 25, 27, 28; histamine, all except 30; nicotinate, all except 17, 20, 21, 26,29 ; trigonelline, $18,23,28$; $n$-dodecane, $16,20,25,26,27,28,30 ; n$-hexadecane, all except $17,18,19,21,22,23,29$.

fucose, glycolate, D-tryptophan, nicotinate, trigonelline, and $n$-dodecane. Considering all the data represented by the symbols in Table 3 , nicotinate and $n$-dodecane were the only compounds for which gross differences were found between the two groups of strains. In most cases where there were differences between the two groups on one basal medium, there were no differences on the other. For most compounds tested there was a higher percentage of $P$. kingae and $P$. multivorans strains positive for growth when tested with the broth medium than with the agar medium. These apparent discrepancies in growth response on agar or broth media are possibly due to differences in the availability of the carbon substrate in agar as opposed to broth media. Another possible explanation might relate to the manner in which growth response was scored on agar media. If growth on a particular substrate was very slight in comparison with a positive control, the plate was scored negative. However, slight turbidity in liquid media was also scored as a negative response. Obviously, there is considerable subjectiveness in growth evaluation for nutritional studies. In our experience, responses with broth media were considerably easier to score and most tubes were either densely turbid or obviously negative compared with controls.

Biochemical, physiological, and morphological characteristics. $P$. kingae and $P$. multivorans had similar biochemical, physiological, and morphological properties (Table 4). The following are tests for which differences were noted: growth at $41 \mathrm{C}$; pigmentation on 
TABLE 4. Comparison of characters of $P$. kingae strains with P. multivorans strains ${ }^{a}$

\begin{tabular}{|c|c|c|}
\hline Character & $P$. kingae $(n=15)$ & P. multivorans $(n=15)$ \\
\hline Denitrification & $-(0)$ & $-(0)$ \\
\hline Nitrate reduction & $\mathrm{V}(47)^{b}$ & $-(13)^{c}$ \\
\hline Gas production in nitrate reduction tests & $-(0)$ & $-(0)$ \\
\hline \multicolumn{3}{|l|}{ Utilization of sole nitrogen sources } \\
\hline Nitrogen & $-(0)$ & $-(0)$ \\
\hline Nitrate & $+(100)$ & $+(100)$ \\
\hline Anaerobic growth in glucose medium of Stanier et al. (30) & $-(0)$ & $-(0)$ \\
\hline Anaerobic growth in $\mathrm{O}-\mathrm{F}$ glucose medium & $-(0)$ & $-(0)$ \\
\hline \multicolumn{3}{|l|}{ Growth in YE broth at: } \\
\hline $4 \mathrm{C}$ & $-(0)$ & $-(0)$ \\
\hline $25 \mathrm{C}$ & $+(100)$ & $+(100)$ \\
\hline $37 \mathrm{C}$ & $+(100)$ & $+(100)$ \\
\hline $39 \mathrm{C}$ & $+(100)$ & $+(100)$ \\
\hline $41 \mathrm{C}$ & $+(93)^{d}$ & $V(60)^{e}$ \\
\hline \multicolumn{3}{|l|}{ Growth on: } \\
\hline MacConkey agar & $+(100)$ & $+(87)^{f}$ \\
\hline Salmonella-Shigella agar & $-(0)$ & $-(7)^{g}$ \\
\hline Simmons' citrate agar & $+(100)$ & $+(100)$ \\
\hline Cetrimide agar & $+(93)^{n}$ & $\mathrm{~V}(40)^{i}$ \\
\hline Pyocyanin, medium A, King et al. (13) & $-(0)$ & $-(0)$ \\
\hline Fluorescein, medium B, King et al. (13) & $-(0)$ & $-(0)$ \\
\hline Yellow pigment on TSI agar & $\mathrm{V}(67)^{j}$ & $-(13)^{k}$ \\
\hline \multicolumn{3}{|l|}{ Other pigments on: } \\
\hline Medium A & $\mathrm{V}(20)^{l}$ & $\mathrm{~V}(80)^{m}$ \\
\hline Medium B & $-(0)$ & $\mathrm{V}(73)^{n}$ \\
\hline YE agar & $+(100)$ & $+(93)^{o}$ \\
\hline Heart infusion tyrosine agar & $-(0)$ & $\mathrm{V}(73)^{p}$ \\
\hline Semisolid medium & $-(0)$ & $\mathrm{V}(80)^{a}$ \\
\hline Intracellular accumulation of poly- $\beta$-hydroxybutyrate & $+(100)$ & $+(100)$ \\
\hline Production of catalase & $+(100)$ & $+(100)$ \\
\hline Production of oxidase & $+(100)$ & $+(100)$ \\
\hline Production of indole & $-(0)$ & $-(0)$ \\
\hline Methyl red test & $-(0)$ & $-(0)$ \\
\hline Production of acetoin (Voges-Proskauer test) & $-(0)$ & $-(0)$ \\
\hline Production of $\mathrm{H}_{2} \mathrm{~S}$ (TSI agar) & $-(0)$ & - $(0)$ \\
\hline Production of opalescence (lecithinase) on egg yolk agar & $\mathrm{V}(80)^{r}$ & $+(100)$ \\
\hline Liquefaction of gelatin, CDC medium (12) & $\mathrm{V}(33)^{s}$ & $\mathrm{~V}(47)^{t}$ \\
\hline Hydrolysis of gelatin, Skerman's medium (26) & $-(7)^{u}$ & $V(47)^{\circ}$ \\
\hline Hydrolysis of urea (Christensen) & $+(100)$ & $+(100)$ \\
\hline Hydrolysis of Tween 80 & $+(100)$ & $+(100)$ \\
\hline Hydrolysis of esculin & $\mathrm{V}(80)^{w}$ & $V(67)^{x}$ \\
\hline Hydrolysis of starch & $-(0)$ & $-(0)$ \\
\hline Peptonization reaction in litmus milk & $+(100)$ & $+(93)^{y}$ \\
\hline Constitutive arginine dihydrolase & $-(0)$ & $-(0)$ \\
\hline ortho cleavage of catechol & $+(100)$ & $+(100)$ \\
\hline Motility & $+(100)$ & $+(93)^{2}$ \\
\hline Multitrichous polar flagellation ( $>1$ flagellum/cell) & $+(100)$ & $+(93)^{a a}$ \\
\hline Oxidative reaction in the Hugh and Leifson O-F test (10) & $+(100)$ & $+(100)$ \\
\hline In peptone broth media, acid from glucose & $\mathrm{V}(60)^{b b}$ & $\mathrm{~V}(60)^{c c}$ \\
\hline $\begin{array}{l}\text { In peptone broth media, acid from xylose, mannitol, lactose, su- } \\
\text { crose and maltose }\end{array}$ & $-(0)$ & $-(0)$ \\
\hline \multicolumn{3}{|l|}{ On O-F medium, acid from: } \\
\hline Glucose & $+(100)$ & $+(100)$ \\
\hline Xylose & $+(93)^{d d}$ & $+(87)^{e e}$ \\
\hline Mannitol & $\mathrm{V}(53)^{\prime \prime}$ & $\mathrm{V}(80)^{\mathrm{gg}}$ \\
\hline Lactose & $+(100)$ & $+(100)$ \\
\hline Sucrose & $+(93)^{n h}$ & $\mathrm{~V}(47)^{i t}$ \\
\hline Maltose & $+(100)$ & $\mathrm{V}(73)^{j j}$ \\
\hline \multicolumn{3}{|l|}{ Acid production from $10 \%(\mathrm{wt} / \mathrm{vol})$ : } \\
\hline glucose agar & $+(100)$ & $+(100)$ \\
\hline lactose agar & $+(100)$ & $+(100)$ \\
\hline
\end{tabular}


a Symbols:,+ 100 to $85 \%$ of strains positive; V, 84 to $16 \%$ of strains positive;,- 15 to $0 \%$ of strains positive. Numbers in parentheses indicate the percentages of strains positive. All percentages were based on a study of 15 strains each of $P$. kingae and $P$. multivorans, except for arginine dihydrolase percentages, which represent 7 strains from each group.

${ }^{b}$ Positive strains: $1,2,3,6,7,9,11$.

${ }^{c}$ Positive strains: 17, 18.

a Negative strain: 11 .

e Negative strains: $16,21,25,27,28,30$.

${ }^{\prime}$ Negative strains: $16,28$.

g Positive strain: 20.

${ }^{n}$ Negative strain: 11.

'Positive strains: 16, 17, 20, 22, 23, 24.

${ }^{j}$ Negative strains: $1,5,6,9,10$.

${ }^{k}$ Positive strains: $19,23$.

'Positive strains: 7, 13, 15.

${ }^{m}$ Negative strains: $18,21,24$.

${ }^{n}$ Negative strains: 18, 21, 23, 24.

- Negative strain: 18.

${ }^{\circ}$ Negative strains: $18,21,23,24$.

${ }^{a}$ Negative strains: $18,23,24$.

$r$ Negative strains: 4, 13, 15.

${ }^{s}$ Positive strains: $2,3,7,11,13$.

${ }^{t}$ Positive strains: $16,20,22,23,25,28,29$.

${ }^{u}$ Positive strain: 11.

${ }^{\circ}$ Positive strains: $16,20,25,27,28,29,30$.

${ }^{w}$ Negative strains: $4,8,15$.

${ }^{x}$ Negative strains: $17,18,26,27,30$.

${ }^{y}$ Negative strain: 18 (weakly acid).

"Negative strain: 23.

aa Negative strain: 23.

${ }^{b 0}$ Negative strains: $2,3,5,7,13,15$.

${ }^{c c}$ Negative strains: $16,20,22,28,29,30$.

${ }^{d d}$ Negative strain: 11.

ee Negative strains: 16, 28.

${ }^{\prime \prime}$ Negative strains: $3,4,6,7,8,9,10$.

${ }^{8}$ Negative strains: $17,18,27$.

${ }^{h h}$ Negative strain: 8 .

'i Positive strains: 17, 19, 22, 23, 24, 26, 29.

${ }^{j j}$ Negative strains: 19, 22, 25, 29.

various media (medium $B$, heart infusion tyrosine, TSI, and motility agars); gelatin hydrolysis (Skerman's plated medium; 26); lecithinase production (egg yolk agar); growth on cetrimide agar; nitrate reduction; and acid production from sucrose and maltose in $\mathrm{O}-\mathrm{F}$ basal medium. The difference noted for gelatin hydrolysis on plates of Skerman's medium was not evident when strains were tested for gelatin liquefaction by using the CDC medium. The difference was probably related to the fact that the CDC medium, which showed a higher number of positive strains for $P$. kingae, was incubated for 7 days, whereas Skerman's medium was incubated for only $48 \mathrm{~h}$. In addition, it should be noted that the difference between the two groups in ability to produce lecithinase was minimal ( $20 \%$ difference).

The differences in pigmentation noted are worthy of explanation. P. multivorans (but not $P$. kingae) strains produced a variety of pigments, including greenish-yellow, yellow, pur- ple, and mixtures of these colors on medium $A$ and medium B. Neither group of strains produced fluorescent pigment (fluorescein) on medium B or pyocyanin on medium A. Production of specific colored pigments by $\boldsymbol{P}$. multivorans seemed to be a function of the medium on which it was grown and the incubation temperature. Even the same strain grown on various carbon substrates in a defined mineral base produced a variety of colored pigments. These findings support the conclusion of Stanier et al. (30) that differences in pigmentation from strain to strain in $P$. multivorans are not correlated with differences in other phenotypic characters and, therefore, are devoid of taxonomic significance. Of interest also is the production by all $P$. kingae strains of yellow and/or buff pigments on YE agar. $P$. multivorans, again, produced a variety of pigments on this medium. Jonsson (11) reported that $66.2 \%$ of his $P$. kingae isolates produced yellow pigment only on media containing iron (i.e., TSI agar). In the present study, 
$67 \%$ of $P$. kingae strains produced yellow pigment on TSI agar. Perhaps the production of yellow and/or buff pigments on YE agar is due to the iron content in the standard mineral base or in autolyzed yeast cells.

Colonial morphology and hemolytic reaction. Colonial morphologies for both groups of strains were highly similar. All colonies showed the following characteristics: circular form; convex elevation; entire margin; punctiform $(<1 \mathrm{~mm})$ to $1 \mathrm{~mm}$ in diameter; smooth, glistening surface; translucent to transparent by transmitted light; grayish by reflected light; and butyrous consistency. Five strains of $P$. multivorans differed slightly from this description. Colonies of one of these strains had a yellow coloration. Colonies from four strains had slightly mottled surfaces, slightly undulate margins, and a cream-gray coloration, and were slightly larger in diameter ( 1 to $2 \mathrm{~mm}$ ). All strains of $P$. kingae and $P$. multivorans showed "lysis" (blood cell lysis but not hemoglobin destruction or decolorization [15]) on blood agar under confluent areas of growth but not under

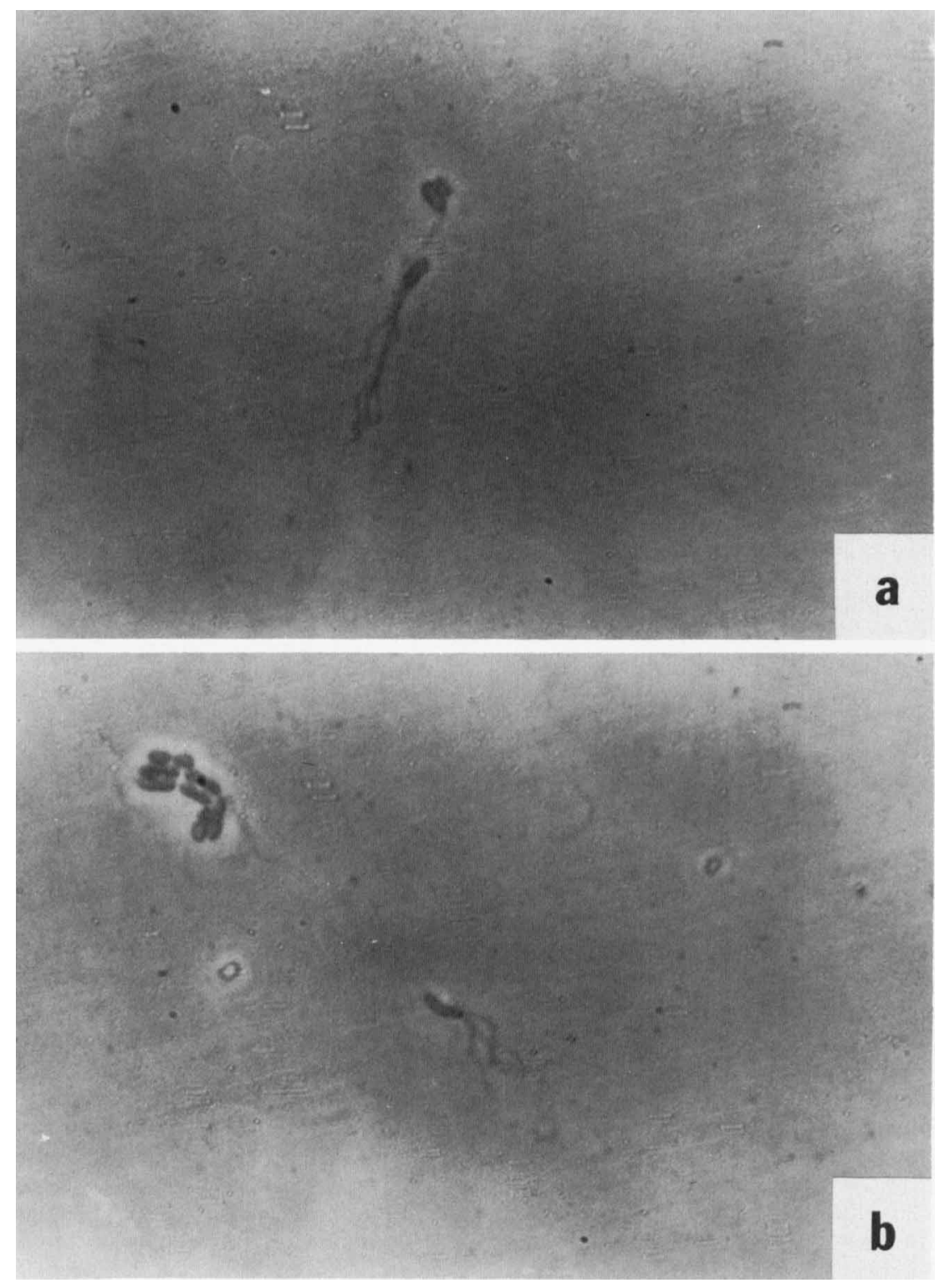

Fig. 1. Phase contrast photomicrographs $(\times 1,500)$ of cells stained for flagella by Leifson's method. $(a) P$. kingae strain no. 1; (b) P. kingae strain no. 8; (c) P. multivorans strain no. 18; (d) P. multivorans strain no. 21 . 
isolated colonies. The edges of "lysis" zones were ill defined. Most $P$. multivorans strains also showed a slight greening of these zones.

Motility and flagella arrangement and number. All strains of $P$. kingae were motile as determined in semisolid medium. All but one strain $(23=$ ATCC 17765) of $P$. multivorans were motile. Flagella-stained preparations showed all strains from both groups to be polar multitrichous, with most cells showing two to five polar flagella (Fig. 1). The only exception was the nonmotile strain of $P$. multivorans, which showed few flagellated cells; some of these cells showed polar flagella and some showed lateral flagella. However, lack of motility and the presence of a small number of laterally flagellated cells was not related to other phenotypic differences. The statistical method of Lautrop and Jessen (16) was applied to evaluate the strains as monotrichous or multitrichous and to demonstrate the similarity in flagella numbers between the two sets of strains. The number of polar flagella was counted on 50 to 100 cells of each strain, and a flagella index was computed by combining the percentages of all flagellated cells possessing more than one
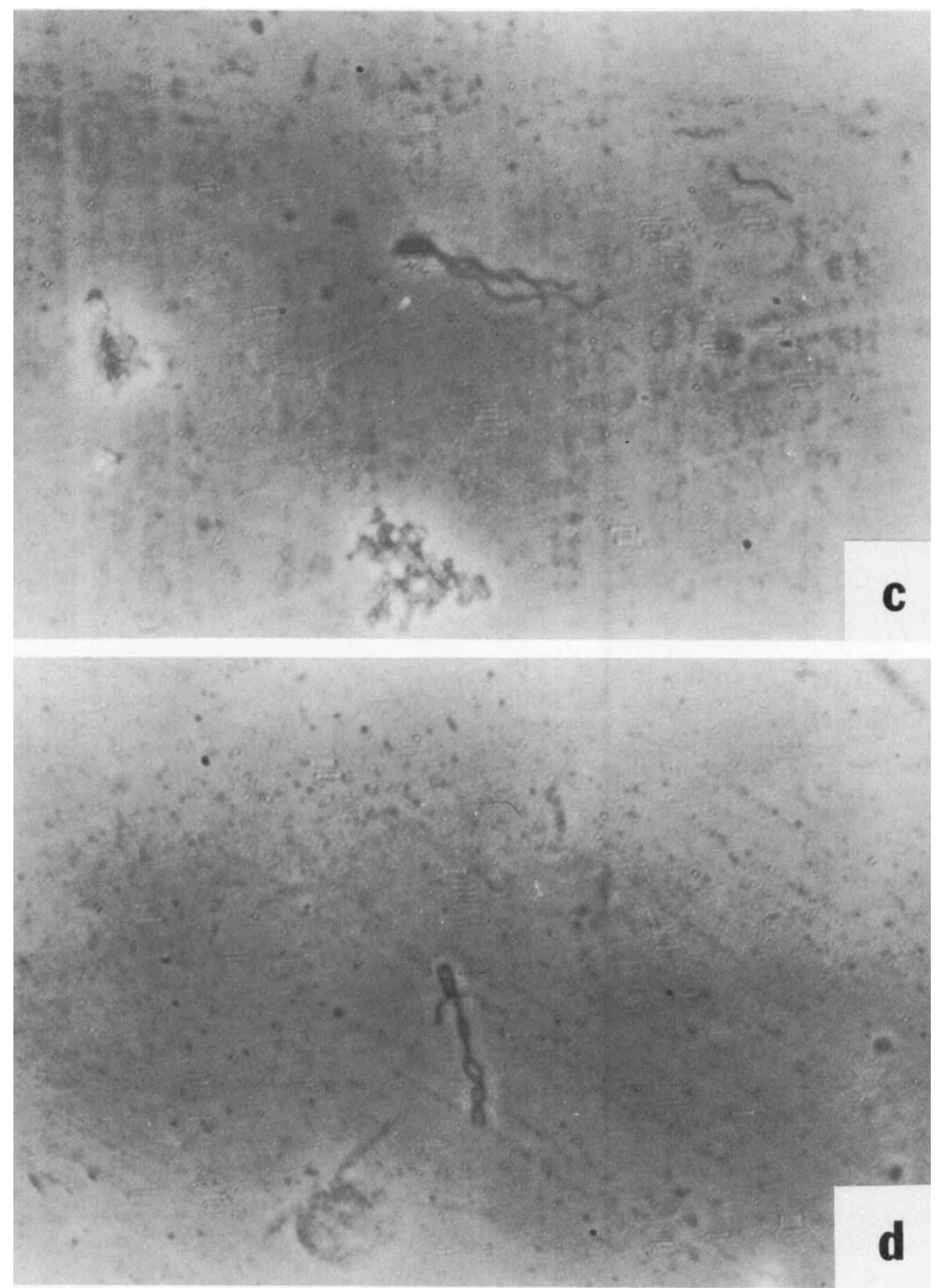

Fig. 1. $C-D$. 
flagellum per pole. The results of these counts (Table 5) showed P. kingae and P. multivorans to be multitrichous and closely similar in flagella numbers and indexes. The computed flagella indexes for $P$. kingae and $P$. multivorans were 64.3 and 69.7 , respectively.

PHB accumulation. Phase microscopy of wet mounts of $P$. kingae and $P$. multivorans grown for $48 \mathrm{~h}$ at $30 \mathrm{C}$ in the basal broth medium $\left[0.02 \%\left(\mathrm{NH}_{4}\right)_{2} \mathrm{SO}_{4}\right]$ with DL- $\beta$-hydroxybutyrate as carbon source showed all strains to contain massive intracellular inclusions of PHB (Fig. 2).

Antibiotic susceptibilities. The antibiotic susceptibilities of $P$. kingae and $P$. multivorans as determined by standardized single-disk diffusion tests are listed in Table 6. P. kingae strains were resistant to 12 of 17 antibiotics tested, whereas $P$. multivorans strains were resistant to 11. Antibiotics for which both groups exhibited variable sensitivities were neomycin, chloramphenicol, kanamycin, gentamicin, and nalidixic acid. In addition, $P$. multivorans showed variable sensitivity to carbenicillin. Considering only differences in all three susceptibility groups, $P$. kingae and $P$. multivorans differed only in their susceptibility to nalidixic acid and carbenicillin. However, the difference in susceptibility to carbenicillin between the two groups of strains was minimal $(27 \%)$.

On the basis of the assigned symbols $(+,-$, V), there was, overall, a $90 \%$ similarity between the 15 strains of $P$. kingae and the 15 strains of $P$. multivorans in response to the 127 tests performed. A total of 26 differences were detected on the basis of these symbols, but $17 \mathrm{can}$ be excluded as not different for the reasons discussed earlier. If these 17 reactions are excluded, then the two groups showed an overall similarity of $94 \%$. A listing of the remaining nine test reactions that differed between the two groups as compared with the reactions found for the type strain of $P$. multivorans is shown in Table 7. Three of these characteristics for $P$. kingae, although different from the composite result for the 15 strains of $P$. multivorans, were the same as those found for the type strain.

In the taxonomic study of Stanier et al. (30), 14 characters were selected that seemed to be of greatest differential value in the recognition of $P$. multivorans. Table 8 shows a comparison of strains of $P$. kingae and $P$. multivorans used in this study with the ideal phenotype of $P$. multivorans based on these 14 selected characters. The strains of $P$. kingae and $P$. multivorans tested in this study were identical when compared on the basis of these 14 characters. The ideal phenotype of Stanier et al. for $P$. multivorans differs from strains of $P$. kingae in the utilization of $\mathrm{D}$-fucose; however, it also differs from the 15 test strains of $P$. multivorans.

On the basis of the similarities in the patterns of the test results, we have concluded that $P$. kingae (EO-1) and $P$. multivorans are indistinguishable, thus confirming the results of Snell et al. (28). The data presented here have extended their studies by inclusion of a larger number of strains and of additional characters not previously used for comparison of these organisms. These additional tests included determination of flagella number and arrangement and antibiotic susceptibility, as well as ability to utilize numerous additional carbon compounds. Ballard et al. (1) and Sands et al. (24) have established that the names $P$. multivorans and $P$. cepacia are synonymous and, according to Snell et al., the name $P$. cepacia has priority over all later synonyms.

TABLE 5. Flagella counts in strains of $P$. kingae and $P$. multivorans

\begin{tabular}{|c|c|c|c|c|c|c|c|c|c|c|c|}
\hline \multirow{2}{*}{ Organism } & \multirow{2}{*}{$\begin{array}{l}\text { No. of } \\
\text { strains }\end{array}$} & \multirow{2}{*}{$\begin{array}{l}\text { Total no. of } \\
\text { cells counted }\end{array}$} & \multicolumn{8}{|c|}{$\begin{array}{l}\text { Percentage of flagellated cells having the } \\
\text { indicated number of flagella }{ }^{b}\end{array}$} & \multirow{2}{*}{$\begin{array}{l}\text { Flagella } \\
\text { index }^{c}\end{array}$} \\
\hline & & & 1 & 2 & 3 & 4 & 5 & 6 & 7 & 8 & \\
\hline P. kingae & 15 & $1,450^{d}$ & 35.7 & 33.9 & 19.5 & 7.4 & 1.9 & 1.1 & 0.2 & 0.3 & 64.3 \\
\hline P. multivorans & 14 & $1,300^{e}$ & 30.3 & 26.7 & 23.8 & 10.8 & 5.0 & 2.1 & 1.1 & 0.2 & 69.7 \\
\hline
\end{tabular}

${ }^{a}$ Unless otherwise indicated, 100 cells/strain were counted.

${ }^{\circ}$ Percentages calculated on the basis of 4 to 8 flagella/cell are approximations.

${ }^{c}$ Combined percentages of all flagellated cells having more than one flagellum/pole.

${ }^{a}$ Strain number $14 ; 50$ cells counted.

e Strain numbers 18 and $26 ; 50$ cells counted. For strain number 23 , no counts were performed because most cells were aflagellate or poorly stained. 

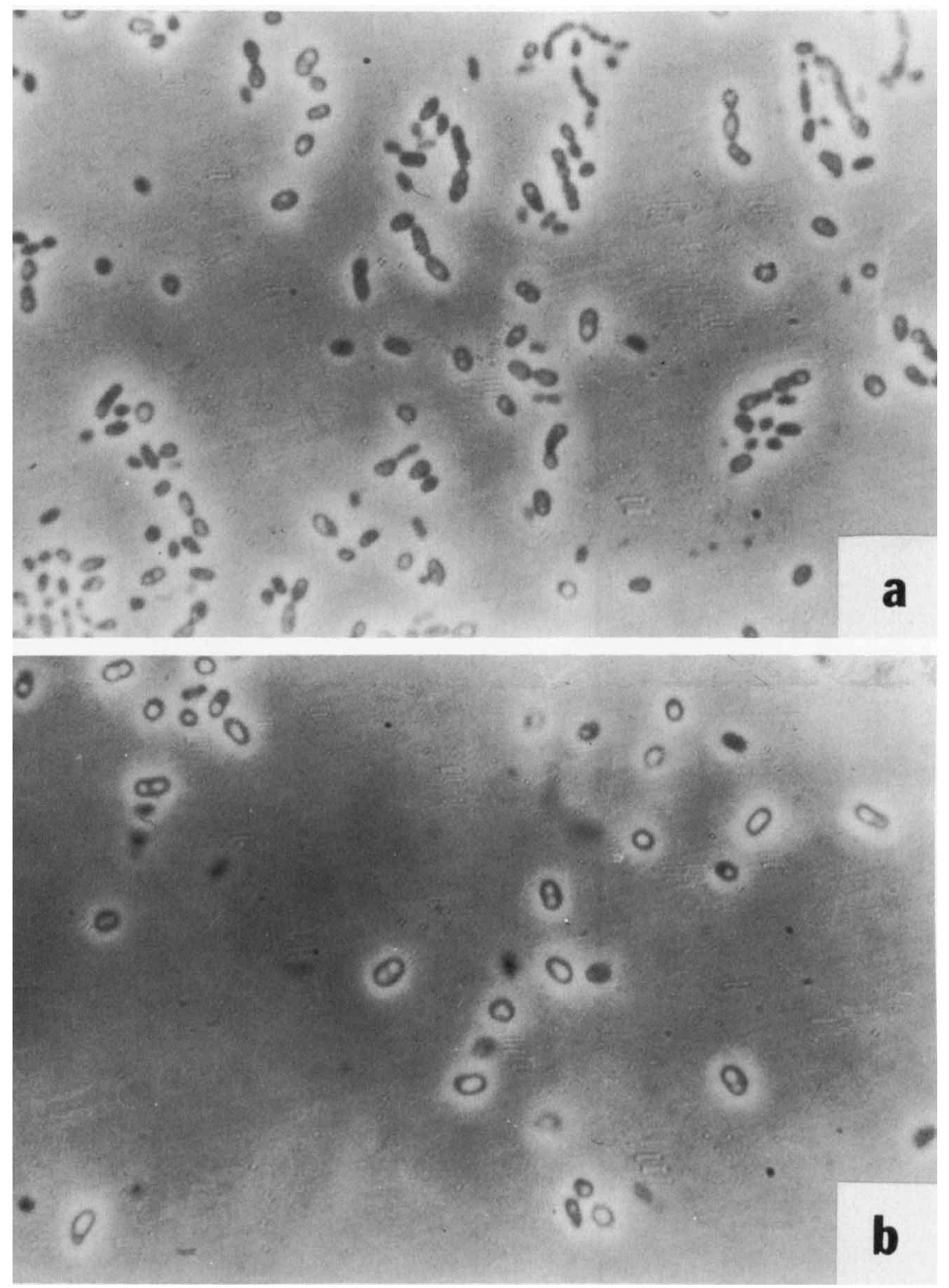

Fig. 2. Phase contrast photomicrographs $(\times 1,500)$ of wet mounts of cells grown for $48 \mathrm{~h}$ in a medium containing DL. $\beta$-hydroxybutyrate. (a) P. kingae strain no. 5; (b) P. kingae strain no. 11; (c) P. multivorans strain no. 16; (d) $P$. multivorans strain no. 29; (e) P. aeruginosa. Note accumulation of poly- $\beta$-hydroxybutyrate as intracellular inclusions in all strains $(a-d)$ except the negative control (e). 

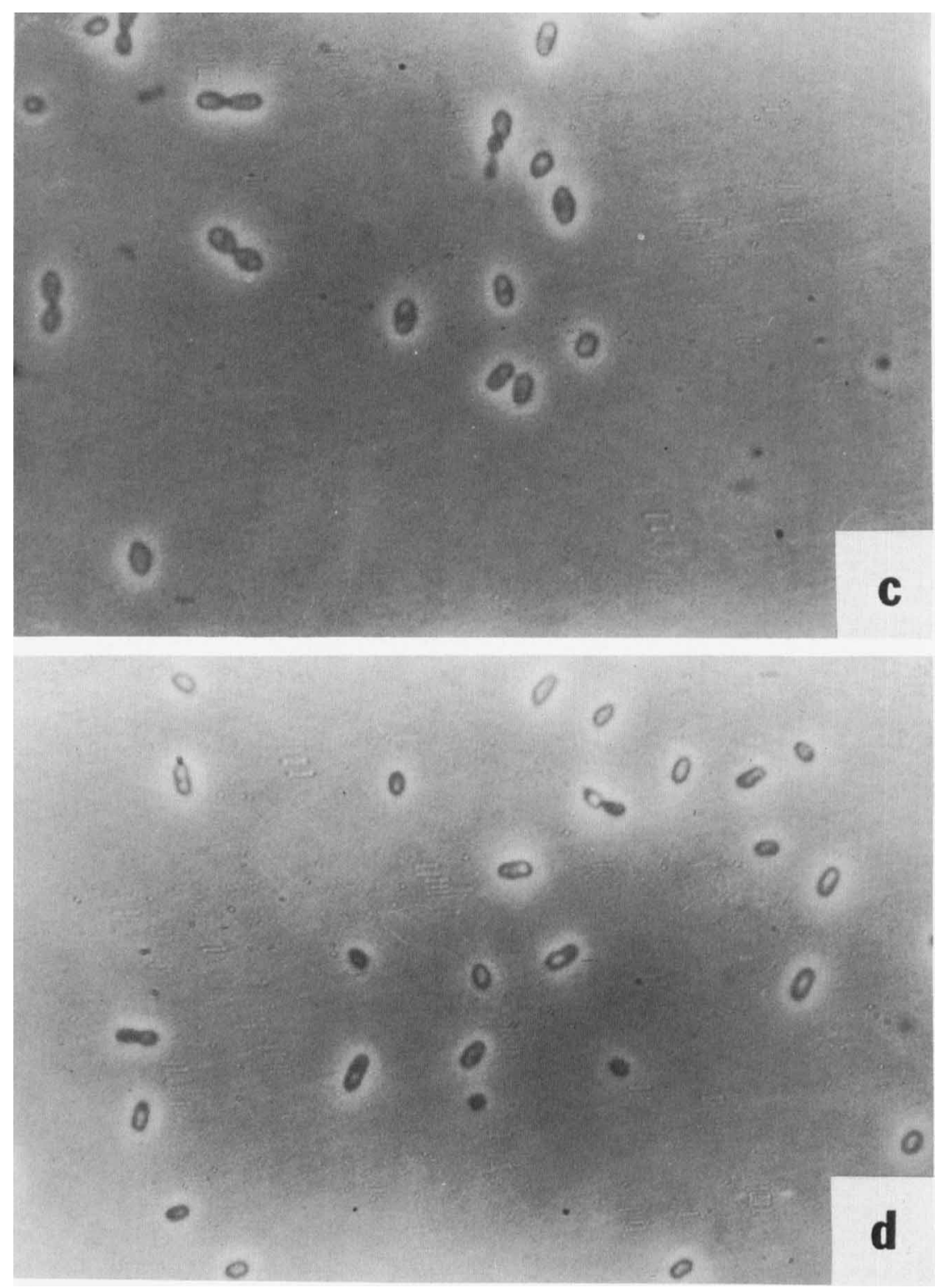

Fig. 2. $C-D$. 


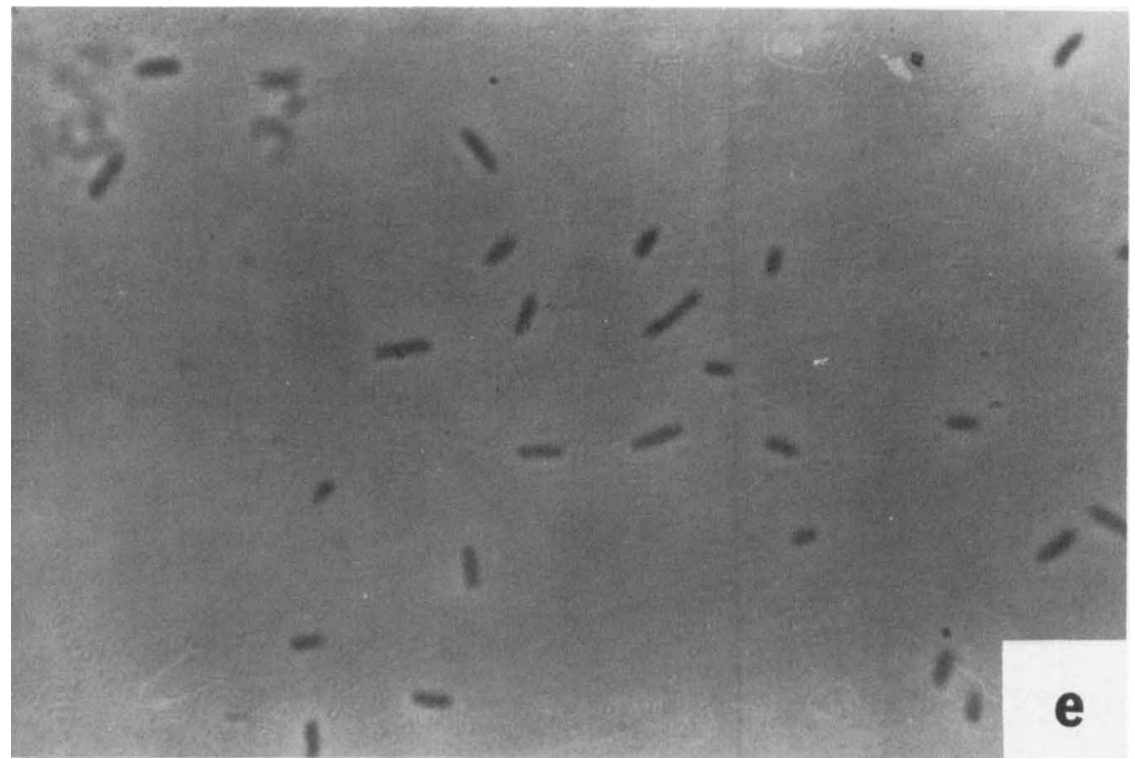

Fig. 2. $E$.

TABle 6. Antibiotic susceptibilities of $P$. kingae and $P$. multivorans strains ${ }^{a}$

\begin{tabular}{|c|c|c|c|c|c|c|c|}
\hline \multirow{2}{*}{ Antibiotic } & \multirow{2}{*}{ Potency ${ }^{\circ}$} & \multicolumn{3}{|c|}{ P. kingae } & \multicolumn{3}{|c|}{ P. multivorans } \\
\hline & & $\mathrm{R}$ & $\mathrm{s}$ & I & $\mathbf{R}$ & $\mathrm{s}$ & I \\
\hline Ampicillin & 10 & $+(100)$ & $-(0)$ & $-(0)$ & $+(93)$ & $-(7)^{c}$ & $-(0)$ \\
\hline Neomycin & 30 & V $(67)$ & $-(13)^{d}$ & $\mathrm{~V}(20)^{e}$ & $\mathrm{~V}(60)$ & $-(13)^{f}$ & $V(27)^{B}$ \\
\hline Chloramphenicol & 30 & $-(0)$ & $V(67)$ & $V(33)^{n}$ & $-(7)^{i}$ & $\mathrm{~V}(73)$ & $\mathrm{V}(20)^{j}$ \\
\hline Kanamycin & 30 & $\mathrm{~V}(60)$ & $V(27)^{k}$ & $-(13)^{t}$ & V $(60)$ & $\mathrm{V}(27)^{m}$ & $-(13)^{n}$ \\
\hline Tetracycline & 30 & $+(100)$ & $-(0)$ & $-(0)$ & $+(93)$ & $-(0)$ & $-(7)^{\circ}$ \\
\hline Gentamicin. & 10 & $V(73)$ & $\mathrm{V}(27)^{p}$ & $-(0)$ & V (67) & $\mathrm{V}(33)^{q}$ & $-(0)$ \\
\hline Nalidixic acid $\ldots \ldots \ldots$ & 30 & $\mathrm{~V}(20)^{r}$ & $\mathrm{~V}(27)^{s}$ & $\mathrm{~V}(53)$ & $\mathrm{V}(27)^{t}$ & $V(60)$ & $-(13)^{4}$ \\
\hline Nitrofurantoin & 300 & $+(100)$ & $-(0)$ & $-(0)$ & $+(100)$ & $-(0)$ & $-(0)$ \\
\hline Lincomycin ... & 2 & $+(100)$ & $-(0)$ & $-(0)$ & $+(100)$ & $-(0)$ & $-(0)$ \\
\hline Colistin ... & 10 & $+(100)$ & $-(0)$ & $-(0)$ & $+(93)$ & $-(7)^{v}$ & $-(0)$ \\
\hline Penicillin G & 10 & $+(100)$ & $-(0)$ & $-(0)$ & $+(100)$ & $-(0)$ & $-(0)$ \\
\hline Methicillin . & 5 & $+(100)$ & $-(0)$ & $-(0)$ & $+(100)$ & $-(0)$ & $-(0)$ \\
\hline Erythromycin & 15 & $+(100)$ & $-(0)$ & $-(0)$ & $+(100)$ & $-(0)$ & $-(0)$ \\
\hline Cephalothin . & 30 & $+(100)$ & $-(0)$ & $-(0)$ & $+(100)$ & $-(0)$ & $-(0)$ \\
\hline Dihydrostreptomycin & 10 & $+(93)$ & $-(0)$ & $-(7)^{w}$ & $+(100)$ & $-(0)$ & $-(0)$ \\
\hline Cephaloglycin ....... & 30 & $+(100)$ & $-(0)$ & $-(0)$ & $+(100)$ & $-(0)$ & $-(0)$ \\
\hline Carbenicillin. & 50 & $+(100)$ & $-(0)$ & $-(0)$ & $\mathrm{V}(73)$ & $\mathrm{V}(27)^{x}$ & $-(0)$ \\
\hline
\end{tabular}

a Abbreviations: R, Resistant; S, susceptible; I, intermediate. Parenthetical values refer to percentages of strains showing indicated susceptibility. For the purpose of comparison, these values have been converted to the following symbols:,+ 100 to $85 \%$; V, 84 to $16 \% ;-, 15$ to $0 \%$.

${ }^{b}$ Disk concentration in micrograms except for penicillin $\mathrm{G}$, which is expressed in international units.

${ }^{c}$ Sensitive strain: 21.

${ }^{d}$ Sensitive strains: 11,13 .

' Intermediate strains: 4, 8, 12 .

'Sensitive strains: 21, 22.

${ }^{8}$ Intermediate strains: $16,18,28,30$.

${ }^{n}$ Intermediate strains: $1,2,11,13,14$.

${ }^{i}$ Resistant strain: 22.

'Intermediate strains: 23, 24, 27.

${ }^{*}$ Sensitive strains: $4,8,11,13$.

'Intermediate strains: $12,15$.

${ }^{m}$. Sensitive strains: $17,18,21,22$.
${ }^{n}$ Intermediate strains: $19,29$.

${ }^{\circ}$ Intermediate strain: 18 .

${ }^{\circ}$ Sensitive strains: $4,8,11,13$.

${ }^{\circ}$ Sensitive strains: $16,18,21,22,28$.

${ }^{r}$ Resistant strains: 6, 9, 13 .

${ }^{s}$ Sensitive strains: $2,4,8,15$.

${ }^{t}$ Resistant strains: 18, 22, 23, 24.

"Intermediate strains: $17,27$.

- Sensitive strain: 21.

"Intermediate strain: 13.

${ }^{x}$ Sensitive strains: $20,21,26,30$. 
TABLE 7. Differences between strains of $P$. kingae and $P$. multivorans compared with data for the type strain of $P$. multivorans ${ }^{a}$

\begin{tabular}{|c|c|c|c|}
\hline Character & $P$. kingae & $\begin{array}{c}P . \text { multi- } \\
\text { vorans }\end{array}$ & $\begin{array}{c}\text { P. multi- } \\
\text { vorans } \\
\text { ATCC } 17759\end{array}$ \\
\hline $\begin{array}{l}\text { Biochemical tests } \\
\text { Growth at } 41 \mathrm{C} \\
\text { Pigmentation } \\
\text { Growth on ce- } \\
\text { trimide agar } \\
\text { Nitrate reduction } \\
\text { On O-F medium, } \\
\text { acid from: } \\
\text { Sucrose } \\
\text { Maltose } \\
\text { Utilization tests } \\
\text { Nicotinate } \\
n \text {-Dodecane } \\
\text { Antibiotic suscepti- } \\
\text { bility } \\
\text { Nalidixic acid }\end{array}$ & $\begin{array}{l}+ \\
- \\
+ \\
\mathrm{v}\end{array}$ & $\begin{array}{l}\text { V } \\
\text { V } \\
\text { V } \\
\text { V }\end{array}$ & $\begin{array}{l}+ \\
+ \\
- \\
-\end{array}$ \\
\hline
\end{tabular}

${ }^{a}$ Symbols: +, Positive; -, negative; V, variable; I, intermediate; $\mathrm{S}$, sensitive.

TABLE 8. Comparison of $P$. kingae and $P$. multivorans on the 14 selected characters of greatest differential value in the recognition of $P$. multivorans ${ }^{a}$

\begin{tabular}{|c|c|c|c|c|}
\hline \multirow[b]{2}{*}{ Character } & \multicolumn{2}{|c|}{ P. multivorans ${ }^{b}$} & \multirow{2}{*}{ P. kingae $e^{c}$} & \multirow[b]{2}{*}{ P. multivorans ${ }^{c}$} \\
\hline & $\begin{array}{c}\text { No. of positive } \\
\text { strains }\end{array}$ & $\begin{array}{c}\text { Ideal } \\
\text { phenotype }\end{array}$ & & \\
\hline 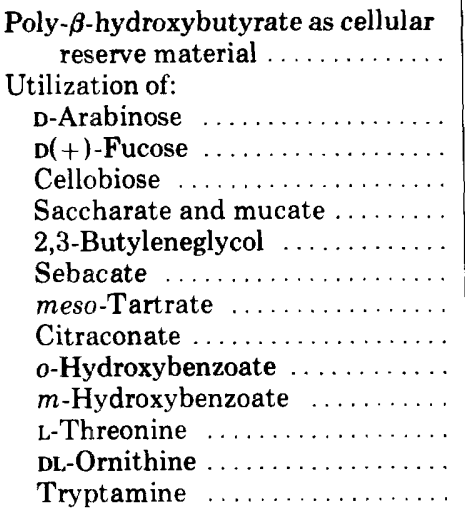 & $\begin{array}{l}19 \\
19 \\
19 \\
19 \\
19 \\
19 \\
19 \\
19 \\
17 \\
14 \\
19 \\
19 \\
18 \\
19\end{array}$ & $\begin{array}{l}+ \\
+ \\
+ \\
+ \\
+ \\
+ \\
+ \\
+ \\
+ \\
+ \\
+ \\
+ \\
+ \\
+\end{array}$ & $\begin{array}{l}+ \\
+ \\
\mathbf{V} \\
+ \\
+ \\
+ \\
+ \\
+ \\
+ \\
+ \\
+ \\
+ \\
+ \\
+\end{array}$ & $\begin{array}{l}+ \\
+ \\
\mathbf{V} \\
+ \\
+ \\
+ \\
+ \\
+ \\
+ \\
+ \\
+ \\
+ \\
+ \\
+\end{array}$ \\
\hline
\end{tabular}

${ }^{a}$ Symbols: + , Positive; V, variable.

${ }^{b}$ Data from the study of Stanier et al. (30) of 19 strains of $P$. multivorans.

${ }^{c}$ Data from the present study based on 15 strains each of $P$. kingae and $P$. multivorans. Substrate utilization tests were performed on agar media except for $o$-hydroxybenzoate and $m$-hydroxybenzoate, which were performed in broth media.

\section{ACKNOWLEDGMENTS}

We gratefully thank R. E. Weaver (CDC) for the P. kingae strains used in this study and Marie Reeves for the statistical evaluation of substrate utilization results on the basal agar medium. We are also grateful to James Macmillan of the Food and Drug Administration and Rutgers University for assistance in the preparation of this manuscript.

\section{REPRINT REQUESTS}

Address reprint requests to: Henry A. Sinsabaugh,
DHEW, Food and Drug Administration, 850 Third Ave., Brooklyn, N.Y. 11232.

\section{LITERATURE CITED}

1. Ballard, R. W., N. J. Palleroni, M. Doudoroff, R. Y. Stanier, and M. Mandel. 1970. Taxonomy of the aerobic pseudomonads: Pseudomonas cepacia, P. marginata, $P$. alliicola and $P$. caryophylli. J. Gen. Microbiol. 60:199-214.

2. Bassett, D. C. J., K. J. Stokes, and W. R. G. Thomas. 
1970. Wound infection with Fseudomonas multivorans. Lancet 1:1188-1191.

3. Bauer, A. W., W. M. M. Kirby, J. C. Sherris, and M. Turck. 1966. Antibiotic susceptibility testing by a standardized single disk method. Am. J. Clin. Pathol. 45:493-496.

4. Burdon, D. W., and J. C. Whitby. 1967. Contamination of hospital disinfectants with Pseudomonas species. $\mathrm{Br}$. Med. J. ii: $153-155$.

5. Burkholder, W. H. 1950. Sour skin, a bacterial rot of onion bulbs. Phytopathology 40:115-117.

6. Cohen-Bazire, G., W. R. Sistrom, and R. Y. Stanier. 1957. Kinetic studies of pigment synthesis by non-sulfur purple bacteria. J. Cell. Comp. Physiol. 49:25-68.

7. Dailey, R. H., and E. J. Benner. 1968. Necrotizing pneumonitis due to the pseudomonad "Eugonic Oxidizer-group 1." N. Engl. J. Med. 279:361-362.

8. Gilardi, G. L. 1970. Characterization of EO-1 strains (Pseudomonas kingii) isolated from clinical specimens and the hospital environment. Appl. Microbiol. 20:521-522.

9. Hardy, P. C., G. M. Ederer, and J. M. Matsen. 1970. Contamination of commercially packed urinary catheter kits with the pseudomonad EO-1. N. Engl. J. Med. 282:33-35.

10. Hugh, R., and E. Leifson. 1953. The taxonomic significance of fermentative versus oxidative metabolism of carbohydrates by various gram negative bacteria. J. Bacteriol. 66:24-26.

11. Jonsson, V. 1970. Proposal of a new species Pseudomonas kingii. Int. J. Syst. Bacteriol. 20:255-257.

12. King, E. O. 1964. The identification of unusual pathogenic gram negative bacteria. National Communicable Disease Center, Atlanta.

13. King, E. O., M. K. Ward, and D. E. Raney. 1954. Two simple media for the demonstration of pyocyanin and fluorescin. J. Lab. Clin. Med. 44:301-307.

14. Kovacs, N. 1956. Identification of Pseudomonas pyocyanea by the oxidase reaction. Nature (London) 178:703.

15. Laboratory methods in special medical bacteriology (course \#8390-C) techniques. 1968. National Communicable Disease Center, Atlanta.

16. Lautrop, H., and O. Jessen. 1964. On the distinction between polar monotrichous and lophotrichous flagellation in green fluorescent pseudomonads. Acta Pathol. Microbiol. Scand. 60:588-598.

17. Lederberg, J., and E. M. Lederberg, 1975 . Replica plating and indirect selection of bacterial mutants. J. Bacteriol. 63:399-406.

18. Leifson, E. 1951. Staining, shape, and arrangement of bacterial flagella. J. Bacteriol. 62:377-389.

19. Mitchell, R. G., and A. C. Hayward. 1966. Postoperative urinary tract infections caused by contaminated irrigating fluid. Lancet 1:793-795.

20. Morris, M. B., and J. B. Roberts. 1959. A group of pseudomonads able to synthesize poly- $\beta$-hydroxybutyric acid. Nature (London) 183:1538-1539.

21. Pedersen, M. M., E. Marso, and M. J. Pickett. 1970. Nonfermentative bacilli associated with man. III. Pathogenicity and antibiotic susceptibility. Am. J. Clin. Pathol. 54:178-192.

22. Pickett, M. J., and M. M. Pedersen. 1970. Characterization of saccharolytic nonfermentative bacteria associated with man. Can. J. Microbiol. 16:351-362.

23. Ryan, K. J., F. D. Schoenknecht, and W. M. M. Kirby. 1970. Disc sensitivity testing. Hosp. Pract. 5:91-100.

24. Sands, D. C., M. N. Schroth, and D. C. Hildebrand. 1970. Taxonomy of phytopathogenic pseudomonads. J. Bacteriol. 101:9-23.

25. Schiff, J., L. S. Suter, R. D. Gourley, and W. D. Sutliff. 1961. Flavobacterium infection as a cause of bacterial endocarditis. Ann. Intern. Med. 55:499-506.

26. Skerman, V. B. D. 1967. A guide to the identification of the genera of bacteria. The Williams \& Wilkins Co., Baltimore.

27. Snedecor, G. W. 1956. Statistical methods. Iowa State University Press, Ames.

28. Snell, J. J. S., L. R. Hill, S. P. Lapage, and M. A. Curtis. 1972. Identification of Pseudomonas cepacia Burkholder and its synonymy with Pseudomonas kingii Jonsson. Int. J. Syst. Bacteriol. 22:127-138.

29. Sorrell, W. B., and L. V. White. 1953. Acute bacterial endocarditis caused by a variant of the genus Herellea. Am. J. Clin. Pathol. 23:134-138.

30. Stanier, R. Y., N. J. Palleroni, and M. Doudoroff. 1966. The aerobic pseudomonads: a taxonomic study. J. Gen. Microbiol. 43:159-271. 